\title{
Great Amount of C.pneumoniae in Ruptured Plaque Vessel Segments at autopsy. A Comparative Study with Stable Plaques
}

\author{
Maria de Lourdes Higuchi, Jussara Bianchi Castelli, Vera D. A iello, Sueli Palomino, Marcia M. Reis, \\ Nadia V. Sambiase, Shizie Fukasawa, Hiram G. Bezerra, José A. F. Ramires
}

São Paulo, SP - Brazil

\begin{abstract}
A possible relationship between $\mathrm{C}$.pneumoniae $(\mathrm{CP})$ infection, atherosclerosis and acute myocardial infarction is a debated matter. Now we performed the search of CP in histological segments offatal ruptured plaques and of stable plaques by histochemistry (Macchiavello stain), immunohistochemistry and in situ hybridization techniques. Electron microscopy and confocal laser microscopy techniques were used in two additional cases. The semi-quantitification of $\mathrm{CP}+$ cells $(0-4+)$ and quantification of lymphocytes demonstrated greater amount of $\mathrm{CP}+$ cells and more inflammation in the adventitia of vulnerable plaque vessel segments than of stable ones, larger amount of $C P+$ cells in adventitia than in the plaque and high frequency of $\mathrm{CP}+$ cells in all groups studied. This preliminary study strongly suggests a direct pathogenetic involvement of adventitial CP in the rupture of the atheromatous plaque, development of acute myocardial infarction and also in the development of atherosclerosis.
\end{abstract}

The relationship between C.pneumoniae (CP), atherosclerosis and acute myocardial infarction was first suggested at the time of an epidemiological study in Finland ${ }^{1}$. Many works have demonstrated by different techniques, the presence of C.pneumoniae in atherosclerotic plaques. However, the reported incidence varies from $0 \%$ to $100 \%^{2-5}$. A clear morphological demonstration of C.pneumoniae was made only in aorta with aneurysm ${ }^{4}$. Such difficulty in demonstrating the C.pneumoniae in coronary specimens led authors to question the relationship between the bacteria, atherosclerosis and plaque instability. Most of the current data indicating such association have come from clinical trials. A pilot trial (ROXIS) using antibiotic therapy suggested the clinical benefits in preventing death and re-infarction for at least 6 months after the initial treatment ${ }^{6}$.

Heart Institute (InCor), University of São Paulo Medical School

Mailing address: Maria de Lourdes Higuchi - InCor - Laboratório de Anatomia Patológica Instituto do Coração - FMUSP - Av. Dr. Enéas C. Aguiar, 44 - 05403-000

- São Paulo - SP - Brazil - e-mail: anplourdes@incor.usp.br.

Received on $13 / 2 / 00$

Accepted on $29 / 2 / 00$
We have previously demonstrated that unstable atheromas are larger than stable ones, and more frequently have positive remodeling ${ }^{7}$. Adventitial inflammation, disappearance of collagen fibrosis and neovascularization are associated with plaque instability and positive remodeling of the vessel ${ }^{8}$. The inflammatory infiltrate in the adventitia is more intense than the inflammation inside the plaque, and this could support the hypothesis that the adventitia may be the main entrance of some infectious agents. We concluded that the unstable plaque is associated with pan-arteritis, which frequently evolve to aneurysmatic vessel enlargement. Such positive vessel remodeling may favour the development of larger fat plaques and plaque instability.

In the present work we looked for C.pneumoniae in the adventitia and in the plaque, using different techniques for detection of C.pneumoniae in situ in unstable and stable plaques in order to clarify whether C.pneumoniae is involved in the etiopathogenesis of such adventitial inflammation in unstable plaques.

\section{Methods}

Three groups of necropsy atheromatous coronary lesions were retrospectively studied: Group A - 11 ruptured thrombosed plaques responsible for fatal acute myocardial infarction from 11 patients; Group B - 11 stable plaques from the same patients of group A, presenting similar grade of obstruction but in another coronary branch; Group C - 11 stable plaque from 11 distinct patients who were submitted to elective by-pass surgery due to stable angina and did not die due to acute myocardial infarction.

Serial paraffin embedded $5 \mu \mathrm{m}$ - thick sections were performed in order to detect $C$.pneumoniae, using 3 techniques- Macchiavellos's method (modified) ${ }^{9}$; Immunohistochemistry (monoclonal antibody, DAKO Co. USA); In situ hybridization (Oligonucleotide probe endlabeled with biotin, synthesized by GIBCO- BRL, USA). The amount of $C$.pneumoniae positive $(\mathrm{CP}+)$ cells was graded in 0 (absence); $1+$ (scarce $\mathrm{CP}+$ cells), $2+$ (moderate number of $\mathrm{CP}+$ cells) and $3+$ (foci with many $\mathrm{CP}+$ cells) and $4+$ (many foci with a lot of CP+ cells), in slides stained by immunohistochemistry technique and Macchiavello's 
method. Both methods demonstrated the same amount of $\mathrm{CP}+$ cells.

Two additional recent cases were also studied by electron microscopy and confocal laser microscopy in order to certify that the positivity for C.pneumonia in macrophages, fibroblasts and smooth muscle cells detected by immunohistochemistry, histochemistry and in situ hibridization were reliable and specific. These two additional cases were from patients who died due to acute myocardial infarction and whose coronary arteries were perfused with formalin. The culprit lesion was detected macroscopically and selected for study through the 5 above described techniques of C.pneumoniae diagnosis. These two cases were not included in the comparative study of the 3 groups previously described.

The quantification of lymphocytes have already been performed in a study with the same cases ${ }^{8}$. These data were used to test the correlation between number of inflammatory cells and score of $\mathrm{CP}+$ cells.

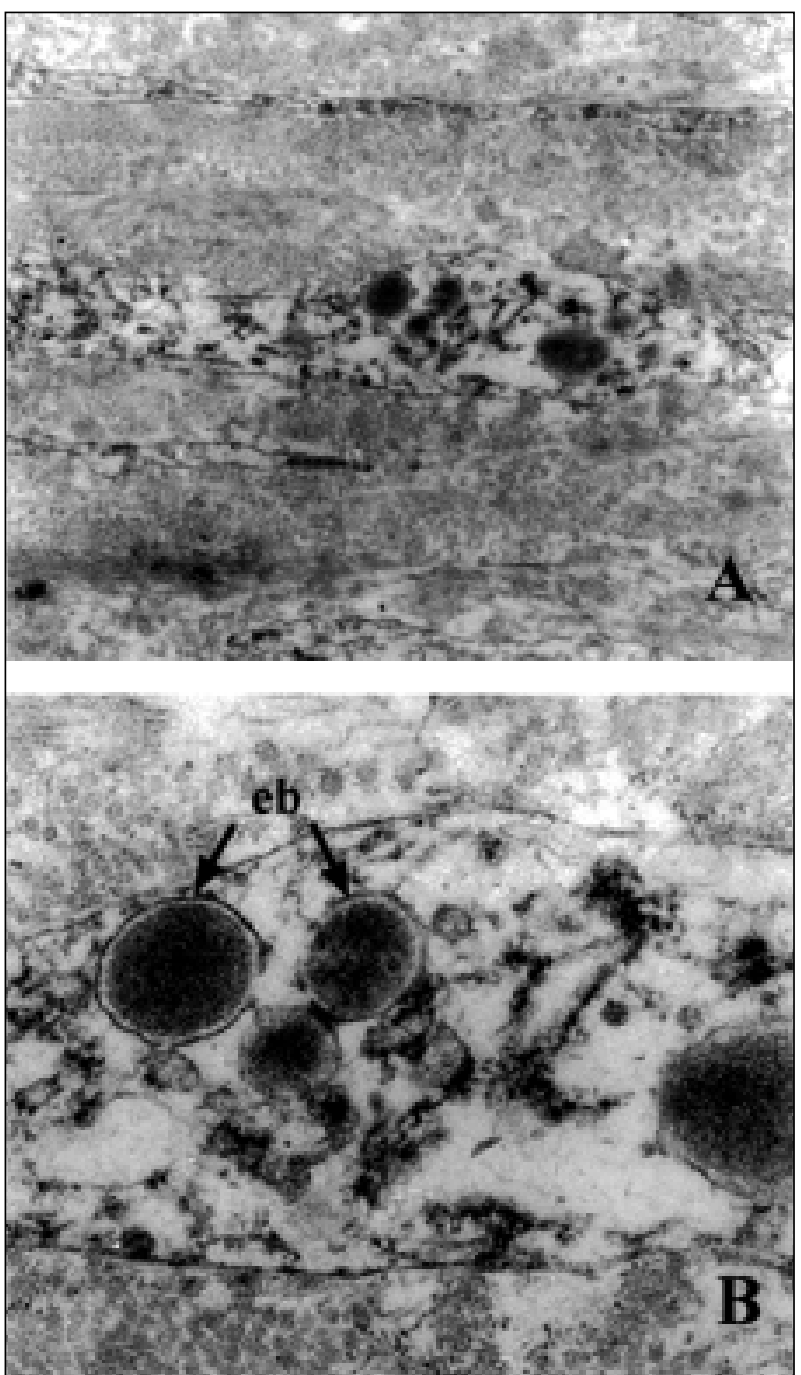

Fig. 1 - Electron micrography of an unstable plaque arterial segment showing one adventitial fibroblast containing many intracellular C.pneumoniae elementary bodies, the infective form of the bacteria (A- original magnification X 4200). A closer view is shown in $\mathrm{B}$; original magnification $\mathrm{X} 10000$.

\section{Results}

All of the techniques showed many fibroblasts and macrophages positive for C.pneumoniae in adventitia (figures 1,2,3,4 and 5), in the adventitia of ruptured thrombosed plaque segments. There were also many positive macrophages at the base of the plaque. The number of positive cells was greater in the adventitia than in the plaque. The frequency of C.pneumoniae detection was very high in groups A, B and C: $100 \% ; 100 \%$ and $82 \%$ and the mean scores of $\mathrm{CP}+$ cells were $2.73 ; 1.55 ; 1.09$ respectively. There was a significantly higher amount of $\mathrm{CP}+$ cells $(\mathrm{t}$ test) in Group A than Group B $(\mathrm{p}<0.005)$ and C $(\mathrm{p}<0.001)$, but no significant difference between Groups B and C. There was no linear correlation between the amount of $\mathrm{CP}+$ cells and number of inflammatory cells. However, there was a significant positive association between high score ( 2 or 3 ) of $\mathrm{CP}+$ cells and moderate or severe $\left(>15\right.$ lymphocytes $\left./ \mathrm{mm}^{2}\right)$ adventitial inflammation $(\mathrm{p}<0,05-$ Chi-2 test).

\section{Discussion}

Most of clinical and epidemiological trials have pointed to an influence of C.pneumoniae in acute myocardial

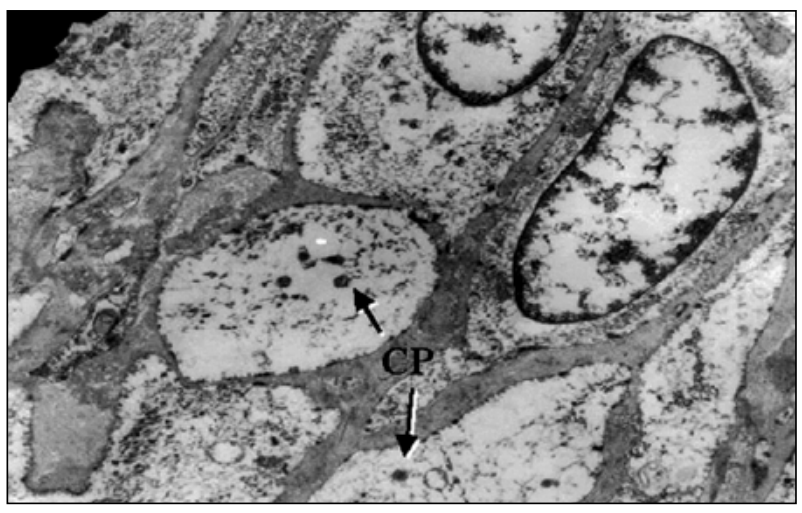

Fig. 2 - Electron micrography of adventitial cells presenting forms of C.pneumoniae (CP-arrows); original magnification X 2600.

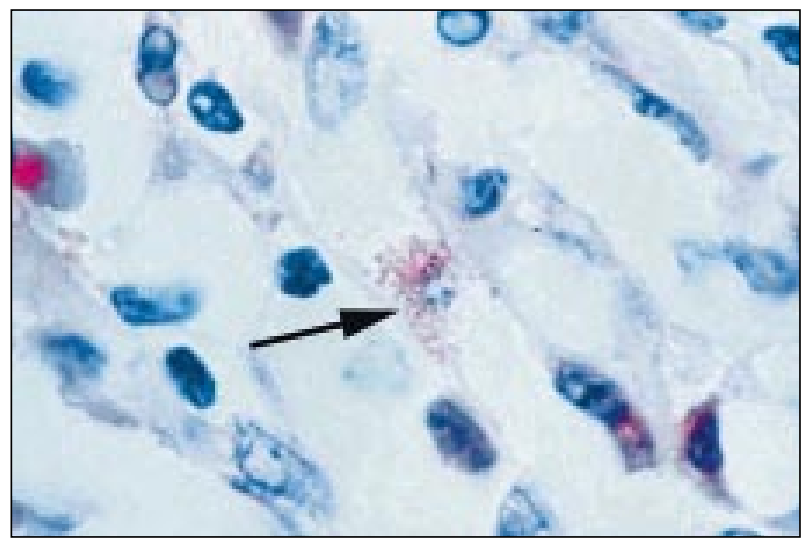

Fig. 3 - Adventitial inflammatory infiltrate from an unstable plaque arterial segment exhibiting a macrophage labeled for C.pneumoniae (arrow) by immunohistochemical reaction (red granules); original magnification X1000. 


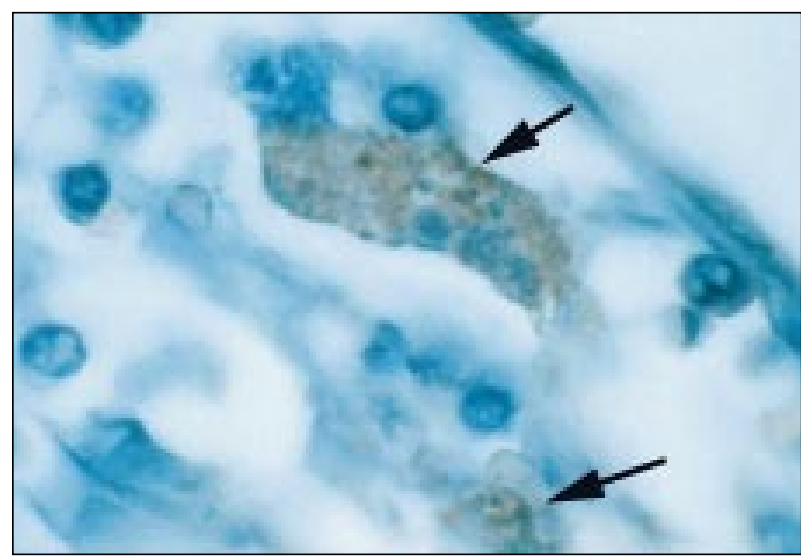

Fig. 4 - Inflammatory infiltrate at the base of the plaque showing macrophages positive for C.pneumoniae (arrows) by in situ hybridization reaction (brownish granules); original magnification $\mathrm{X} 1000$.

infarction or unstable angina. However, the lack of morphological data demonstrating presence of $C$.pneumoniae in situ in the plaque has led many authors to attribute the inflammation in the plaque to autoimmune process, questioning the direct role of the bacteria ${ }^{10}$ or pointing to an indirect action of it by heat shock protein pathway ${ }^{11}$ in the development of plaque instability.

Other authors have recently demonstrated CP membrane protein positivity by immunohistochemistry not only in the atheromatous plaque but also in the adventitia from old patients ${ }^{12}$. In the present work, we reported for the first time a significantly larger amount of C.pneumoniae + cells in ruptured fatal thrombosed plaques than unstable ones. Moreover, we could demonstrate by electron microscopy
C. pneumoniae in ruptured plaque vessel segments at autopsy

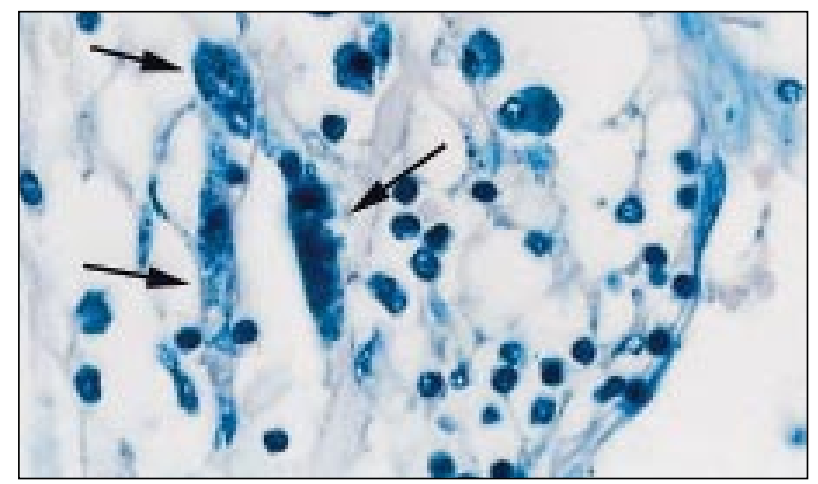

Fig. 5 - Adventitial inflammatory infiltrate from an unstable plaque arterial segment stained by Macchiavello's method, showing macrophages with cytoplasmic granules (arrows); original magnification X 630 .

that the intact bacteria (not only their fragments)are present mainly in the adventitial layer. Moderate to severe inflammation is associated with high numbers of parasitized cells. These results strongly favour the concept that C.pneumoniae is directly involved in the development of adventitial and plaque inflammation (pan-arteritis), leading to plaque rupture. A high frequency of $C$.pneumoniae in all groups suggests its involvement in the pathogenesis of atherosclerosis.

Conclusion - Fatal vulnerable atheromatous plaques are associated with larger amount of C.pneumoniae + cells and severe inflammation in the plaque and adventitia, strongly suggesting a direct pathogenetic involvement of C.pneumoniae in the rupture of the atheromatous plaque and development of acute myocardial infarction.

\section{References}

1. Saikku PLM, Matilla K, Elkman MR, et al. Serologic evidence of an association of a novel Chlamydia TWAR with coronary artery disease and acute myocardial infarction. Lancet 1988; 2: 983-6.

2. Weiss SM, Roblin PM, Gaydos CA, et al. Failure to detect Chlamydia pneumoniae in coronary atheromas of patients undergoing atherectomy. J Infect Dis 1996; 173: 957-62.

3. Paterson DL, Hall J, Rasmussen SJ, Timms P. Failure to detect C.pneumoniae in atherosclerotic plaques of Australian patients. Pathology 1998; 30: 169-72.

4. Juvonen J, Juvonen T, Laurila A, et al. Demonstration of Chlamydia pneumoniae in the walls of abdominal aortic aneurysms. J Vasc Surg 1997; 25: 499-505.

5. Campbell LA, O'Brien ER, Cappucio AL, et al. Isolation of Chlamydia pneumoniae from the coronary artery of a patient with coronary atherosclerosis. The Chlamydia pneumoniae/Atherosclerosis Study Group. Ann Intern Med 1996; 125: 979-82.

6. GurfinkelE, Bozovich G, BeckE, Testa E, Livellara B, Mautner B. Treatment with antibiotic roxithromycin in patients with acute non-Q-waved coronary syndromes. The final report of the ROXIS Study. Eur Heart J 1999; 20: 121-7.

7. Bezerra HG, Higuchi ML, Palomino S, Silvestre J, Gutierrez PS, Ramires JAF
Atheromas that cause fatal thrombosis are larger and have greater compensatory enlargement than equi-stenotic plaques in the same coronary tree. Circulation 1999; 100: I251.

8. Higuchi ML, Bezerra HG, Palomino S, Aiello VD, Libby P, Ramires JAF. Adventitial fibrosis and inflammation surrounding atheroma: Implications for different arterial remodeling in stable and unstable plaques. J Am Coll Cardiol 2000; 35 (2 suppl A): 368A

9. Culling CFA, Allison RT, Barr WT. Microorganisms. In: Culling CFA, Allison RT, BarrWT, Eds. Cellular Pathology Technique. $4^{\text {th }}$ Ed. London: Butterworths \& Co., 1985: 344.

10. de Boer OJ, van Der Wal AC, Becker AE. Atherosclerosis, inflammation and infection. J Pathol 2000; 190: 237-43.

11. Kol A, Sukhova GK, Lichtman AH, Libby P. Chlamydial heat shock protein 60 localizes in human atheroma and regulates macrophage tumor necrosis factoralpha and matrix metalloproteinase expression. Circulation 1998; 98: 300-7.

12. Vink A, Pasterkamp G, Poppen M, Schoneveld AH, Kleijn DPV, Roholl PJM. The adventitia of atherosclerotic coronary arteries frequently contains Chlamydia pneumoniae. J Am Coll Cardiol 2000; 35 (2suppl A): 312A 